\title{
Tác động của các nhân tố bên trong doanh nghiệp đến chất lượng thông tin báo cáo tài chính trong các doanh nghiệp tại Việt Nam
}

\author{
Phạm Quốc Thuần
}

\begin{abstract}
Tóm tắt—Nghiên cứu này hướng đến việc xây dựng thang đo, đo lường chất lượng thông tin (CLTT) báo cáo tài chính (BCTC) theo phương pháp trực tiếp dựa trên các thuộc tính CLTT BCTC được ban hành bởi Hội đồng tiểu chuẩn kế toán tài chính Hoa kỳ (FASB) và Ử ban chuẩn mực kế toán quốc tế (IASB) vào năm 2010 (FASB \& IASB 2010). Bên cạnh đó, bằng nghiên cứu định tính, nghiên cứu đã xác định 5 nhân tố bên trong doanh nghiệp (DN) tác động đến thuộc tính Thích đáng (Relevance) của CLTT BCTC là Hành vi quản trị lọi nhuận (QTLN); Hỗ trọ̣ từ phía nhà quản trị (HTNQT); Đào tạo và bồi dưỡng (ĐTvBD); Hiệu quả của Hệ thống kiểm soát nội bộ (HQKSNB); Năng lực nhân viên kế toán (NLNVKT). Bằng nghiên cứu định lượng, nghiên cứu đã xây dựng mô hình hồi quy cho thấy mức độ tác động của nhân tố QTLN; HTNQT; ĐTvBD; NLNVKT đến tính Thích đáng của CLTT BCTC.
\end{abstract}

Tù khóa-Thích đáng, chất lượng thông tin, báo cáo tài chính, Hành vi quản trị lợi nhuận, Hỗ trợ từ phía nhà quản trị, Đào tạo và bồi dưỡng, Hiệu quả của Hệ thống kiểm soát nội bộ, Năng lực nhẩn viên kế toán

\section{GIỚI THIÊU CHUNG}

$\mathrm{T}$ HÔNG tin kế toán được xem là thành phần chính yếu của thông tin quản lý, đảm nhận vai trò quản lý nguồn lực thông tin tài chính cho các $\mathrm{DN}$, thu thập và ghi nhận các nghiệp vụ kinh tế tài chính phát sinh tại các $\mathrm{DN}$ và cung cấp các thông tin kinh tế, tài chính phục vụ cho nhu cầu của người sử dụng $[4 ; 8]$. Joseph cho rằng thông tin kế toán nghèo nàn sẽ là một đe dọa cho khả năng cạnh tranh của các tổ chức [9]. Người sử dụng thông tin luôn phải đối mặt với việc tiếp nhận những thông tin trên $\mathrm{BCTC}$ kém tin cậy từ những

Ngày nhận bản thảo: 07-02-2018, ngày chấp nhận đăng: 0205-2018, ngày đăng: 15-7-2018.

Tác giả Phạm Quốc Thuần, công tác tại trường Đại học Kinh tế - Luật, ĐHQG HCM (e-mail: thuanpq@uel.edu.vn). nguyên nhân khác nhau như sự khó khăn trong việc tiếp cận nguồn thông tin; sự đối mặt với các động cơ gian lận thông tin từ phía đối tượng cung cấp thông tin; từ những nguyên nhân phát sinh trực tiếp từ bản chất của nghiệp vụ và quá trình kinh doanh. Sự gian lận thông tin kế toán đã gây nên nhiều tác hại cho chính DN lẫn cho xã hội. Xuất phát từ thực trạng trên, tác giả cho rằng CLTT trong đó có CLTT BCTC cũng như các nhân tố ảnh hưởng đến nó là một trong những vấn đề được xã hội và các DN quan tâm hiện nay.

Vấn đề cốt lõi của nghiên cứu về CLTT BCTC là tìm hiểu phương thức xác định và đánh giá CLTT BCTC [3]. Tổng quan các nghiên cứu trước cho thấy đa phần việc đo lường CLTT BCTC thường được thực hiện theo phương pháp gián tiếp (thông qua đánh giá $\mathrm{CL}$ của lợi nhuận trên $\mathrm{BCTC}$ để suy ra kết luận về $\mathrm{CL}$ của $\mathrm{BCTC}$; đánh giá mối quan hệ giữa số liệu lợi nhuận trên $\mathrm{BCTC}$ với phản ứng của thị trường chứng khoán, qua đó đo lường tính Thích hợp của thông tin trên $\mathrm{BCTC}, \ldots$ ) và còn rất ít nghiên cứu tiến hành đo lường CLTT $\mathrm{BCTC}$ theo phương pháp trực tiếp (đo lường CLTT BCTC căn cứ trên các thuộc tính về CLTT). Bên cạnh đó, các nhân tố trong mô hình nghiên cứu còn rời rạc chưa được tập trung vì vậy khó có thể so sánh mức độ tác động giữa các nhân tố đến CLTT BCTC $[5 ; 1]$.

Nghiên cứu này được thực hiện nhằm đo lường CLTT BCTC theo phương pháp trực tiếp; xác định, đo lường các nhân tố tác động đến CLTT BCTC trong các DN tại Việt Nam; Xây dựng mô hình hồi quy phản ánh mức độ tác động của các nhân tố đến CLTT BCTC. Các thành phần đo lường CLTT $\mathrm{BCTC}$ và các nhân tố tác động đến nó rất đa dạng, trong khuôn khổ hạn hẹp của bài viết này, tác giả tiếp cận CLTT BCTC ở khía cạnh thuộc tính Thích đáng theo quan điểm của (FASB \& IASB 2010 và chỉ xem xét các nhân tố tác động 
từ phía bên trong DN. Kết quả nghiên cứu sẽ cung cấp những thông tin định lượng về tính Thích đáng của CLTT BCTC trong các DN Việt Nam hiện nay cũng như khẳng định rõ mức độ tác động của các nhân tố bên ngoài $\mathrm{DN}$ đến nó, từ đó tạo cơ sở cho việc đánh giá thực trạng về CLTT BCTC trên khía cạnh Thích đáng cũng như gợi ý một số chính sách, giải pháp nhằm gia tăng CLTT $\mathrm{BCTC}$ của các DN tại Việt Nam..

\section{CƠ SỞ LÝ THUYÊT}

\subsection{Các khái niệm chính yếu}

Khái niệm CLTT BCTC: FASB (1993) khái niệm CLTT BCTC được xác định bằng các thuộc tính Thích đáng [2], Đáng tin cậy và Dễ hiểu cho người dùng; IASB [7] cho rằng $\mathrm{CL}$ được hiểu như là những thuộc tính làm cho những thông tin trình bày trên các $\mathrm{BCTC}$ trở nên hữu ích đối với những người sử dụng thông tin (Dễ hiểu; Thích đáng; Đáng tin cậy; Có thể so sánh); theo quan điểm hài hòa giữa IASB \& FASB, CLTT BCTC được xác định thông qua thuộc tính hữu ích bao hàm Thích đáng, Trình bày trung thực được nâng cao bởi tính Có thể so sánh, Có thể kiểm chứng, Kịp thời và Dễ hiểu [7]. Trong phạm vi bài viết, tác giả khái niệm CLTT BCTC dựa trên quan điểm hài hòa của IASB \& FASB, theo đó CLTT BCTC được đo lường bằng ba thuộc chính chính yếu: Thích đáng, Trình bày trung thực và các thuộc tính làm gia tăng CLTT BCTC.

Khái niệm Thích đáng: Thích đáng được khái niệm là khả năng tác động của thông tin đến việc ra quyết định của người sử dụng. Thông tin có thể có khả năng ảnh hưởng đến việc ra quyết định khi nó mang giá trị dự đoán, giá trị xác nhận hay cả hai [7], trong đó:

+ Giá trị dự đoán: thông tin được sử dụng như là một thông tin đầu vào bởi người sử dụng khi dự đoán các kêt quả tương lai. [7]

+ Giá trị xác nhận: thông tin cung cấp những phản hồi về việc thừa nhận hoặc những sự thay đổi của các đánh giá trước đó. [7]

\subsection{Co sở lý thuyết}

Mối quan hệ giữa các biến độc lập với CLTT BCTC trong mô hình nghiên cứu được biện luận và lý giải căn cứ trên nền tảng các lý thuyết cơ sở như: Lý thuyết đại diện (Hành vi quản trị lợi nhuận; Hiệu quả của HTKSNB); Quan điểm về Chất lượng tổng thể- TQM (Hỗ trợ từ phía nhà quản trị; Năng lực nhân viên; Đào tạo và bồi dưỡng). Nền tảng của các lý thuyết cơ sở trên giúp hình thành cơ sở vững chắc cho nội dung ở phần nghiên cứu định tính và hình thành nên các giả thuyết nghiên cứu.

\subsection{Tổng quan các nghiên cúu trước}

Hongjiang $\mathrm{Xu} \&$ ctg đã tiến hành nghiên cứu tìm hiểu các nhân tố tác động đến CLTT BCTC trong các $\mathrm{DN}$ tại Australia [5]. Nghiên cứu này được tiến hành thông qua nhiều bước. Đầu tiên nhóm tác giả đã tham khảo cơ sở lý thuyết và các nghiên cứu trước đó thuộc lĩnh vực thông tin quản lý để rút ra các nhóm nhân tố tác động đến CLTT. Sau đó các nhóm nhân tố này được kiểm định lại bằng nghiên cứu định tính thông qua nghiên cứu tình huống. Kết quả phân tích từ nghiên cứu tình huống cho thấy các nhóm nhân tố về Con người (Đào tạo và huấn luyện; Vai trò của con người trong kiểm soát), Hệ thống (Tương tác giữa con người với hệ thống; Vai trò của hệ thống kiểm soát), Tổ chức (Cấp trúc, Văn hóa tổ chức) và Nhân tố bên ngoài (sự thay đổi công nghệ, chính sách,...) thực sự tác động đến CLTT BCTC. Céline Michailesco đã tiến hành tìm hiểu các nhân tố tác động đến CLTT BCTC của các DN Pháp trong khoản thời gian từ 1991-1995 [1]. Nghiên cứu của Céline Michailesco (2010) được tiến hành bằng khảo sát trên $100 \mathrm{DN}$ tại Pháp có thực hiện niêm yết trên thị trường chứng khoán liên tục trong 5 năm. Sau đó, tác giả đã tiến hành phân tích dữ liệu qua hai giai đoạn: phân tích mô tả định lượng để kiểm tra các mối liên hệ của các nhân tố được đưa ra từ giả thuyết nghiên cứu và phân tích khẳng định các mối quan hệ giữa các nhân tố thông qua mô hình hồi quy trên. Kết quả nghiên cứu cho thấy mô hình có $\mathrm{R} 2$ giao động từ $10,82 \%$ đến $22,13 \%$ tùy theo các năm. Xét trên tổng thể thời gian nghiên cứu (1991-1995) chỉ có hai yếu tố tác động đến CLTT BCTC là yếu tố niêm yết trên thị trường chứng khoán trong nước và yếu tố niêm yết trên nhiều thị trường chứng khoán. Sự phân bố quyền sở hữu vốn tác động đến CLTT vào các năm 1993-1995; mức độ nợ chỉ tác động đến CLTT vào năm 1995.

Trên cơ sở tổng hợp các nghiên cứu trước, tác giả thấy rằng các nhân tố tác động đến CLTT BCTC khá đa dạng và được phân làm hai nhóm nhân tố chính: bên trong $\mathrm{DN}$ (Đào tạo và bồi dưỡng; Vai trò của nhà quản trị cấp cao,...) và bên ngoài DN (Niêm Yết; vai trò của Kiểm toán độc lập,...). Tuy nhiên những nghiên cứu về chủ đề 
này còn khá rời rạc, chưa hình thành nên mô hình nghiên cứu với đầy đủ các nhân tố. Bên cạnh đó, đối tượng của các nghiên cứu trước đa phần là $\mathrm{CL}$ hệ thống thông tin kế toán hoặc CLTT kế toán nói chung. Vì vậy nghiên cứu định tính được tác giả lựa chọn để xác định các nhân tố cho mô hình nghiên cứu.

\section{PHƯƠNG PHÁP NGHIÊN CÚUU VÀ XÂY DỨNG THANG ĐO}

Dựa trên mục tiêu nghiên cứu đã được xác định, phần nghiên cứu định tính giúp xác định, xây dựng thang đo đo lường các nhân tố tác động đến tính Thích đáng của CLTT BCTC. Nghiên cứu định lượng giúp đo lường các nhân tố và kiểm định, xác định mức độ tác động của các nhân tố bên trong $\mathrm{DN}$ đến tính Thích đáng của CLTT BCTC.

\subsection{Nghiên cứu định tính}

Nghiên cứu định tính được tiến hành bằng nghiên cứu tình huống với các đối tượng tham gia thảo luận bao gồm Kế toán trưởng; Trưởng bộ phận IT; Giám đốc tài chính; Trưởng phòng KSNB; Trưởng phòng kiểm toán. Với số lượng mẫu được xác định theo phương pháp bảo hòa với 14 tình huống, kết quả phân tích cho thấy có 5 nhân tố bên trong $\mathrm{DN}$ được cho là tác động đến CLTT BCTC: QTLN, HTNQT, ĐTvBD, HQKSNB, NLNVKT.

\subsection{Mô hình, giả thuyết nghiên cưu}

Dựa trên kết quả của nghiên cứu định tính, tác giả xây dựng mô hình cho nghiên cứu theo Hình 1 .

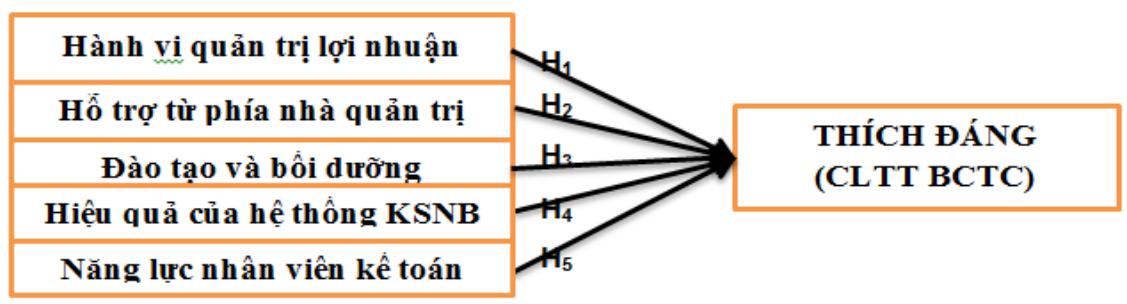

Hình 1. Mô hình nghiên cứu (nguồn: xây dựng từ kết quả nghiên cứu)

Dựa trên mục tiêu nghiên cứu đã được xác định, phần nghiên cứu định tính giúp xác định, xây dựng thang đo đo lường các nhân tố tác động đến tính Thích đáng của CLTT BCTC. Nghiên cứu định lượng giúp đo lường các nhân tố và kiểm định.

\subsection{Thang do}

Nguyên tắc xây dựng thang đo: tác giả sử dụng những thang đo đã có sẵn sau khi điều chỉnh cho phù hợp với bối cảnh nghiên cứu tại Việt Nam thông qua các ý kiến rút ra nghiên cứu tình huống. Đối với những khái niệm mới, thang đo được xây dựng dựa trên ý kiến thu thập từ nghiên cứu tình huống. Về cấp bậc, nghiên cứu sử dụng thang đo cấp quãng, cụ thể là thang đo Likert với năm mức độ.

Đo lường Tính thích đáng của CLTT BCTC: Thích đáng là một thang đo bậc 2 bao gồm hai thành phần: Giá trị dự đoán; Giá trị xác nhận. Giá trị dự đoán được đo lường bởi 3 biến quan sát: (1) Thông tin trên BCTC của đơn vị đủ tin cậy để nhà đầu tư ra quyết định- DĐ1; (2) Thông tin trên $\mathrm{BCTC}$ của đơn vị đủ tin cậy để người cho vay, chủ nợ ra quyết định- DĐ2; (3) Thông tin trên BCTC của đơn vị đủ tin cậy để tiên đoán các kết quả tương lai-DĐ3. Giá trị xác nhận được đo lường bởi 3 biến quan sát: (1) Thông tin trên $\mathrm{BCTC}$ đủ tin cậy để đánh giá tình hình thực hiện kế hoạch của DN- XN1MH; (2) Thông tin trên BCTC không đủ tin cậy để đánh giá hiệu quả hoạt động của DN- XN2MH; (3) Thông tin trên BCTC phản ánh chưa xác đáng thực trạng kinh tế, tài chính của $\mathrm{DN}-\mathrm{XN} 3 \mathrm{MH}$.

Thang đo QTLN: là thang đo bậc 1 bao gồm 4 biến quan sát: Ý muốn của nhà QT có ảnh hưởng đến việc xử lý và trình bày BCTC-QTLN1; Định hướng của nhà $\mathrm{QT}$ có tác động đến việc lựa chọn phương pháp kế toán-QTLN2; Nhà QT thường xuyên tác động đến công việc kế toán tại đơn vịQTLN3; Nhà QT thường yêu cầu xử lý TT kế toán theo ý của mình-QTLN4.

Thang đo HTNQT: là thang đo bậc 1 bao gồm 4 biến quan sát: Nhà quản trị luôn đánh giá cao tầm quan trọng của CLTT kế toán-QTLN1; Thiết kế \& vận hành hệ thống thông tin kế toán luôn có sự tham gia của nhà quản trị-QTLN2; Nhà quản trị luôn cung cấp đủ nguồn lực cho tổ chức và vận 
hành HT TTKT-QTLN3; Nhà quản trị luôn yêu cầu việc trình bày TT kế toán phải trung thực, hợp lý-QTLN4.

Thang đo ĐTvBD: là thang đo bậc 1 bao gồm 4 biến quan sát: Đơn vị luôn yêu cầu nhân viên phải luôn nâng cao trình độ về kế toán và thuếĐTvBD1; Đơn vị luôn hỗ trợ việc đào tạo và bồi dưỡng kiến thức về $\mathrm{KT}$, thuế- ĐTvBD1; Đơn vị luôn có chế độ đào tạo và bồi dưỡng nhân viên khi cập nhật, nâng cấp hệ thống thông tin kế toánĐTvBD1; Đơn vị có kế hoạch và thực hiện đào tạo và bồi dưỡng liên tục- ĐTvBD1.

Thang đo HQKSNB: là thang đo bậc 1 bao gồm 4 biến quan sát: đơn vị có thiết lập các quy định và thủ tục kiểm soát nhằm đảm bảo $\mathrm{DN}$ tuân thủ pháp luật-HQKSNB1; KSNB tại đơn vị giúp ngăn ngừa hiệu quả các gian lận và sai sótHQKSNB2; KSNB tại đơn vị giám sát thường xuyên hoạt động của hệ thống thông tin kế toán kế toán-HQKSNB3; KSNB luôn kiểm tra và giám sát việc đảm bảo CLTT kế toán-HQKSNB4.

Thang đo NLNVKT: là thang đo bậc 1 bao gồm 4 biến quan sát: nhân viên kế toán chưa am hiểu rõ chuẩn mực \& chế độ KT-NLKTMH1; Khả năng hiểu \& vận dụng quy định kế toán vào thực tế của nhân viên còn hạn chế-NLKTMH1; nhân viên nhân viên chưa am hiểu rõ quy trình, đặc điểm SXKD của đơn vị-NLKTMH1; nhân viên kế toán chưa am hiểu rõ bản chất nghiệp vụ kinh tế của đơn vị-NLKTMH1.

\subsection{Nghiên cứu định lượng}

Nghiên cứu này được tiến hành bằng phương pháp khảo sát với đối tượng thu thập dữ liệu chính yếu là Kế toán trưởng tại các $\mathrm{DN}$ có trụ sở chính tại TPHCM và các tỉnh lân cận như Bình Dương, Đồng Nai, Long An,.... Để đánh giá độ tin cậy của thang đo, nghiên cứu dùng hệ số Cronbach alpha; để kiểm định giá trị thang đo, nghiên cứu tiến hành phân tích nhân tố khám phá EFA [10]. Để kiểm định giả thuyết nguyên cứu và xác định mức độ tác độ của các nhân tố trong mô hình, tác giả dùng phương pháp phân tích hồi quy.

\section{KÊTT QUẢ NGHIÊN CÚU}

\subsection{Mô tả mẫu nghiên cưu}

Nghiên cứu được thực hiện trên một mẫu bao gồm $180 \mathrm{DN}$. Trong số này, có $137 \mathrm{DN}$ chưa niêm yết, 21 niêm yết tại Việt Nam và 22 niêm yết ở nước ngoài. Về kiểm toán độc lập, có $53 \mathrm{DN}$ chưa thực hiện kiểm toán $\mathrm{BCTC} ; 70$ được ngoài Big 4 kiểm toán và 57 được big4 kiểm toán. Về trụ sở chính, có $145 \mathrm{DN}$ có trụ sở chính tại TP. $\mathrm{HCM}$, số còn lại tại các tỉnh lân cận như Đồng Nai, Bình Dương, Long An,...

\subsection{Thực trạng về tính Thích đáng của CLTT BCTC trong các DN Việt Nam}

Dựa trên giá trị của các biến quan sát thu thập được qua quá trình khảo sát, tác giả tiến hành tính toán giá trị Thích đáng, và của các thành phần đo lường nó (Giá trị xác nhận và Giá trị dự đoán) trên cơ sở lấy giá trị trung bình của các biến (Bảng 1).

BẢNG 1

ĐO LƯỜNG TÍNH THÍCH ĐÁNG CỦA CLTT BCTC

\begin{tabular}{lrrrrrrr}
\hline \hline & \multicolumn{2}{c}{$\mathbf{N}$} & & & & & \\
& \multicolumn{1}{c}{ Tổng quan sát } & Quan sát bị lỗi & Trung bình & Trung vị & Mode & Độ lệch chuẩn & Phương sai \\
\cline { 2 - 5 } THICHDANG & 180 & 0 & 3,7231 & 4,0000 & 4,00 & 0,84235 & 0,710 \\
GTDĐ & 180 & 0 & 3,7370 & 4,0000 & 4,00 & 0,77033 & 0,593 \\
GTXN & 180 & 0 & 3,7093 & 4,0000 & 4,00 & 1,05293 & 1,109 \\
\hline \hline
\end{tabular}

Nguồn: số liệu khảo sát thực tế 2015 và được kết xuất tù phần mềm SPSS.

Kết quả phân tích theo Bảng 1 cho thấy nếu xét theo khía cạnh Thích đáng thì CLTT BCTC trong các DN Việt Nam hiện nay được đánh giá theo mức độ khá $(3,72 / 5$ điểm). Điều này cho thấy $\mathrm{BCTC}$ trong các $\mathrm{DN}$ hiện nay vẫn chưa đảm bảo độ tin cậy ở mức cao để các đối tượng sử dụng ra quyết định và tiên đoán các kết quả tương lai của DN cũng như phản ánh chưa thật xác đáng toàn bộ quá trình hoạt động kinh doanh của $\mathrm{DN}$.

\subsection{Mô tả mẫu nghiên cứu}

Kết quả kiểm định cho thấy Cronbach $\propto$ của các thang đo đều có giá trị cao (từ 0,818 đến 0,939 ) và hệ số tương quan biến tổng của các biến đều trong khoản $0,608-0,917(>0,3)$ cho thấy các thành phần thang đo đo lường khái niệm nghiên cứu có độ tin cậy cao và các biến quan sát đều đạt yêu cầu (xem Bảng 2). 
BẢNG 2

KÊT QUẢ PHÂN TÍCH CRONBACH $\alpha$ CHO CÁC THANG ĐO

\begin{tabular}{|c|c|c|c|c|}
\hline $\begin{array}{l}\text { Biến quan } \\
\text { sát }\end{array}$ & $\begin{array}{c}\text { Trung bình thang đo nếu } \\
\text { loại biến }\end{array}$ & $\begin{array}{c}\text { Phương sai thang đo nếu } \\
\text { loại biến }\end{array}$ & $\begin{array}{c}\text { Tương quan } \\
\text { biến tồng }\end{array}$ & $\begin{array}{c}\text { Cronbach's Alpha nếu } \\
\text { loại biến }\end{array}$ \\
\hline \multicolumn{5}{|c|}{ Thành phần: Giá trị dự đoán (GTDĐ), Alpha = 0,870 } \\
\hline DĐ1 & 7,45000 & 2,428 & 0,799 & 0,772 \\
\hline DĐ2 & 7,32778 & 2,501 & 0,781 & 0,790 \\
\hline DĐ3 & 7,64444 & 2,655 & 0,677 & 0,884 \\
\hline \multicolumn{5}{|c|}{ Thành phần: Giá trị xác nhận(GTXN), Alpha = 0,907 } \\
\hline $\mathrm{XN1MH}$ & 7,48889 & 4,676 & 0,781 & 0,894 \\
\hline $\mathrm{XN} 2 \mathrm{MH}$ & 7,31667 & 4,642 & 0,855 & 0,833 \\
\hline $\mathrm{XN} 3 \mathrm{MH}$ & 7,45000 & 4,606 & 0,807 & 0,872 \\
\hline \multicolumn{5}{|c|}{ Thành phần: Hành vi quản trị lọi nhuận (QTLN), Alpha = 0,890 } \\
\hline QTLN1 & 9,14444 & 8,783 & ,786 & ,849 \\
\hline QTLN2 & 9,12222 & 9,337 & 689 & ,885 \\
\hline QTLN3 & 9,51111 & 8,542 & ,797 & 845 \\
\hline QTLN4 & 9,80556 & 8,761 & ,766 & ,856 \\
\hline \multicolumn{5}{|c|}{ Thành phần: Hỗ trọ̣ từ phía nhà quản trị (HTNQT), Alpha = 0,818 } \\
\hline HTNQT1 & 11,72778 & 4,568 & ,720 & ,737 \\
\hline HTNQT2 & 11,95000 & 4,506 & 608 & ,786 \\
\hline HTNQT3 & 11,88889 & 4,714 & ,609 & ,784 \\
\hline HTNQT4 & 11,88333 & 4,439 & ,629 & ,776 \\
\hline \multicolumn{5}{|c|}{ Thành phần: Đào tạo và bồi dưỡng (ĐTvBD), Alpha = 0,939 } \\
\hline ĐTvBD1 & 11,36111 & 7,953 & ,780 & ,944 \\
\hline ĐTvBD2 & 11,61667 & 6,886 & ,917 & ,900 \\
\hline ĐTvBD3 & 11,63333 & 7,060 & ,887 & ,910 \\
\hline ĐTvBD4 & 11,82222 & 6,661 &, 853 & ,924 \\
\hline \multicolumn{5}{|c|}{ Thành phần: Hiệu quả của Hệ thống kiểm soát nội bộ (HQKSNB), Alpha = 0,932 } \\
\hline HQKSNB1 & 10,96111 & 7,736 & ,736 & ,944 \\
\hline HQKSNB2 & 10,97778 & 7,474 & 840 & ,911 \\
\hline HQKSNB3 & 11,06667 & 7,068 & 899 & 891 \\
\hline HQKSNB4 & 11,09444 & 7,237 & ,888 & ,895 \\
\hline \multicolumn{5}{|c|}{ Thành phần: Năng lực nhân viên kế toán (NLNVKT), Alpha = 0,927 } \\
\hline NLKTMH1 & 11,46111 & 6,853 & 823 & ,908 \\
\hline NLKTMH2 & 11,53889 & 7,043 &, 842 & ,900 \\
\hline NLKTMH3 & 11,22778 & 7,439 & 840 & ,902 \\
\hline NLKTMH4 & 11,18889 & 7,350 &, 817 & ,908 \\
\hline
\end{tabular}

4.4 Kiểm định giá trị thang đo bằng phân tích EFA

Kiểm định mức độ quan hệ giũa các biến đo luờng

Có nhiều tiêu chí để đánh giá mối quan hệ giữa các biến (Nguyễn Đình Thọ, 2011), trong đó có phép kiểm định Bartlett (Barlette có $\mathrm{p}<5 \%$ ); Kiểm định $\mathrm{KMO}(\mathrm{KMO}>0,50)$. Kết quả kiểm định Bartlett và $\mathrm{KMO}$ cho thang đo Thích đáng thuộc CLTT BCTC (biến phụ thuộc) và thang đo các nhân tố tác động đến CLTT BCTC (biến độc lập) cho thấy $\mathrm{p}=0,000<5 \%$ và $\mathrm{KMO}>0,50$ (Bảng 3 ) như vậy thang đo được xem là phù hợp để phân tích EFA. 
BẢNG 3

KIỂM ĐỊNH BARTLETT VÀ KMO (BẢNG 3 )

\begin{tabular}{lcc}
\hline \hline \multicolumn{1}{c}{ Kiểm định KMO và Bartlett } & KMO & $\begin{array}{c}\text { Giá trị } \\
\text { Sig. }\end{array}$ \\
\hline $\begin{array}{l}\text { Thang đo biến phụ thuộc } \\
\text { Thang đo biến độc lập }\end{array}$ & 0,862 & 0,000 \\
\hline \hline
\end{tabular}

Nguồn: số liệu khảo sát thục tế 2015 và được kết xuất tù̀ phần mềm SPSS

Phân tích EFA cho thang đo thuộc biến phu thuộc (Thích đáng)

Trong nghiên cứu của mình, tác giả giả thuyết ban đầu là thang đo Thích đáng có 2 thành phần: Giá trị dự đoán; Giá trị xác nhận. Để phân tích EFA cho thang đo này, đầu tiên tác giả tiến hành xem xét nhân tố trích cho 2 thành phần trên. Kết quả phân tích theo Bảng 4 cho thấy có 2 nhân tố trích được với tổng phương sai trích TVE là $82,1 \%$. Điều này cho thấy thang đo đạt giá trị phân biệt. Tác giả tiếp tục xem xét ma trận trọng số nhân tố dựa vào phép trích PCA để xem xét các biến sẽ được nhóm vào nhân tố nào (Bảng 5 ). Kết quả phân tích cho thấy trọng số nhân tố của các biến đều được nhóm vào các biến mà nó đo lường và đều $>0,50$ cho thấy các biến này thực sự đo lường khái niệm chúng ta cần đo lường. Các kết quả trên cho thấy thang đo này đạt giá trị hội tụ.

BẢNG 4

NHÂN TỐ VÀ PHƯƠNG SAI TRÍCH CỦA THANG ĐO THÍCH ĐÁNG CỦA CLTT BCTC

\begin{tabular}{|c|c|c|c|c|c|c|c|c|c|}
\hline \multicolumn{10}{|c|}{ "Tống phương sai giải thích (Total Variance Explained) } \\
\hline \multirow[b]{2}{*}{ Nhân tố } & \multicolumn{3}{|c|}{ Giá trị Eigen ban đầu } & \multicolumn{3}{|c|}{ Tổng bình phương tải trọng trích được } & \multicolumn{3}{|c|}{ Tổng bình phương tải trọng xoay } \\
\hline & $\mathrm{TC}$ & \% phương sai & Lũy kế \% & $\mathrm{TC}$ & $\%$ phương sai & Lũy kế \% & $\mathrm{TC}$ & $\%$ phương sai & Lũy kế \% \\
\hline$\overline{1}$ & 4,181 & 69,684 & 69,684 & 4,181 & 69,684 & 69,684 & 2,529 & 42,154 & 42,154 \\
\hline 2 & 0,743 & 12,385 & 82,068 & 0,743 & 12,385 & 82,068 & 2,395 & 39,915 & 82,068 \\
\hline 3 & 0,406 & 6,770 & 88,839 & & & & & & \\
\hline 4 & 0,290 & 4,832 & 93,670 & & & & & & \\
\hline 5 & 0,212 & 3,533 & 97,203 & & & & & & \\
\hline 6 & 0,168 & 2,797 & 100,000 & & & & & & \\
\hline
\end{tabular}

Phương pháp trích: Principal Component Analysis.

Nguồn: số liệu khảo sát thực tế 2015 và được kết xuất tì̀ phần mềm SPSS.

BẢNG 5

MA TRẬN TRỌNG SỐ NHÂN TỐ THANG ĐO THÍCH ĐÁNG CỦA CLTT BCTC

\begin{tabular}{lcr}
\hline \hline & Ma trận trọng số nhân tố đã xoay (Rotated Component Matrix) & \\
\cline { 2 - 3 } & \multicolumn{2}{c}{ Nhân tố $^{\mathbf{a}}$} \\
\cline { 2 - 4 } DĐ1 & Giá trị xác nhận & Giá trị dụ̣ đoán \\
DĐ2 & 0,279 & $\mathbf{0 , 8 8 8}$ \\
DĐ3 & 0,376 & $\mathbf{0 , 8 2 8}$ \\
XN1MH & 0,370 & $\mathbf{0 , 7 5 1}$ \\
XN2MH & $\mathbf{0 , 8 4 6}$ & 0,316 \\
XN3MH & $\mathbf{0 , 8 6 5}$ & 0,363 \\
PNy & $\mathbf{0 , 8 4 2}$ & 0,354 \\
\hline
\end{tabular}

Phương pháp trích: Principal Component Analysis.

Phương pháp xoay: Varimax with Kaiser Normalization

Nguồn: số liệu khảo sát thực tế 2015 và được kết xuất tì̀ phần mềm SPSS.

Phân tích EFA cho thang đo thuộc biến độc lập

Tác giả giả thuyết ban đầu là có 5 nhân tố thuộc biến độc lập. Kết quả phân tích (Bảng 6) cho thấy có 5 nhân tố trích được tại Eigenvalues là 1,248 với tổng phương sai trích TVE là 79,583\%. Điều này hoàn toàn phù hợp với giả thuyết ban đầu của tác giả đề ra. Tiếp theo, kiểm định giá trị hội tụ của thang đo này dựa vào phép trích $\mathrm{PCA}$ cho thấy các trọng số nhân tố của biến đều được nhóm vào các biến mà nó đo lường và đều $>0,50$ cho thấy các biến này thực sự đo lường khái niệm chúng ta cần đo lường (Bảng 7). 
SCIENCE \& TECHNOLOGY DEVELOPMENT JOURNAL:

ECONOMICS - LAW AND MANAGEMENT, VOL 2, NO 1, 2018

BẢNG 6

NHÂN TỐ VÀ PHƯƠNG SAI TRÍCH CỦA THANG ĐO THUỘC BIÊN ĐỘC LẬP

\begin{tabular}{|c|c|c|c|c|c|c|c|c|c|}
\hline \multicolumn{10}{|c|}{ Tổng phương sai giải thích (Total Variance Explained) } \\
\hline \multirow[b]{3}{*}{ Nhân tố } & \multicolumn{3}{|c|}{ Giá trị Eigen ban đầu } & \multicolumn{3}{|c|}{ Tổng bình phương tải trọng trích được } & \multicolumn{3}{|c|}{ Tổng bình phương tải trọng xoay } \\
\hline & & $\%$ & & & $\%$ & & & & \\
\hline & $\mathrm{TC}$ & phương sai & Lũy kế \% & $\mathrm{TC}$ & phương sai & Lũy kế \% & $\mathrm{TC}$ & \% phương sai & Lũy kế \% \\
\hline 1 & 7,770 & 38,851 & 38,851 & 7,770 & 38,851 & 38,851 & 3,436 & 17,179 & 17,179 \\
\hline 2 & 3,126 & 15,630 & 54,481 & 3,126 & 15,630 & 54,481 & 3,341 & 16,704 & 33,883 \\
\hline 3 & 2,179 & 10,895 & 65,376 & 2,179 & 10,895 & 65,376 & 3,285 & 16,423 & 50,306 \\
\hline 4 & 1,593 & 7,966 & 73,342 & 1,593 & 7,966 & 73,342 & 3,117 & 15,584 & 65,891 \\
\hline 5 & 1,248 & 6,241 & 79,583 & 1,248 & 6,241 & 79,583 & 2,738 & 13,692 & 79,583 \\
\hline 6 & ,581 & 2,907 & 82,489 & & & & & & \\
\hline 7 &, 533 & 2,665 & 85,154 & & & & & & \\
\hline 8 & ,427 & 2,135 & 87,289 & & & & & & \\
\hline 9 & ,380 & 1,899 & 89,188 & & & & & & \\
\hline$\ldots$ & $\ldots$ & $\ldots$ & $\ldots$ & & & & & & \\
\hline
\end{tabular}

Phương pháp trích: Principal Component Analysis.

Nguồn: số liệu khảo sát thực tế 2015 và được kết xuất tì̀ phẩn mểm SPSS.

BẢNG 7

MA TRẬN TRỌNG SỐ NHÂN TỐ THANG ĐO CÁC BIẾN ĐỘC LẬP

\begin{tabular}{|c|c|c|c|c|c|}
\hline & \multicolumn{5}{|c|}{ Ma trận trọng số nhân tố đã xoay ${ }^{a}$} \\
\hline & ĐT\&BD & NLNVKT & HQKSNB & QTLN & HTNQT \\
\hline QTLN1 &,- 048 &,- 124 &,- 123 & ,866 &,- 101 \\
\hline QTLN2 & ,049 &,- 136 &,- 137 & ,808 &, 121 \\
\hline QTLN3 &,- 081 &,- 177 &,- 162 & ,854 &,- 037 \\
\hline QTLN4 &,- 156 &,- 287 &,- 155 & ,794 &,- 173 \\
\hline HTNQT1 &, 183 &, 140 &, 100 &,- 099 &, 822 \\
\hline HTNQT2 & ,086 &, 078 &, 107 & ,136 & ,798 \\
\hline HTNQT3 &, 277 &, 041 &, 126 &,- 024 & ,725 \\
\hline HTNQT4 &, 182 &, 129 & ,289 &,- 260 & ,686 \\
\hline ĐTvBD1 & ,848 &, 110 &, 185 &,- 051 &, 125 \\
\hline ĐTvBD2 & ,878 & ,135 &, 230 &,- 086 & ,242 \\
\hline ĐTvBD3 & ,836 & ,133 &, 323 &,- 078 & ,223 \\
\hline ĐTvBD4 & ,841 & ,134 & ,252 &,- 013 & ,214 \\
\hline HQKSNB1 & , 130 & , 102 & ,754 &,- 197 &, 332 \\
\hline HQKSNB2 & ,349 & ,082 &, 805 &,- 189 & , 157 \\
\hline HQKSNB3 & ,281 & ,068 & ,894 &,- 124 &, 114 \\
\hline HQKSNB4 & ,271 & ,092 & ,881 &,- 174 & ,096 \\
\hline NLKTMH1 & ,042 & ,890 & ,149 &,- 123 & ,033 \\
\hline NLKTMH2 & ,112 & ,883 & ,013 &,- 197 & ,074 \\
\hline NLKTMH3 & , 149 & ,866 &, 064 &,- 185 & ,153 \\
\hline NLKTMH4 & , 156 &, 855 & ,076 &,- 182 & ,128 \\
\hline \multicolumn{6}{|c|}{ Phương pháp trích: Principal Component Analysis. } \\
\hline
\end{tabular}




\subsection{Kết quả phân tích hồi quy}

Kiểm tra giả định liên hệ tuyến tính: tác giả dùng Biểu đồ Scatter để kiểm định giả định liên hệ tuyến tính (Hình 2). Rất dễ dàng để quan sát được là phần dư được phân tán ngẫu nhiên trong một vùng xung quanh đường đi qua tung độ 0 . Điều này cho thấy phần dư và giá trị dự đoán đã được chuẩn hóa là độc lập nhau, giả định liên hệ tuyến tính được thỏa mãn.

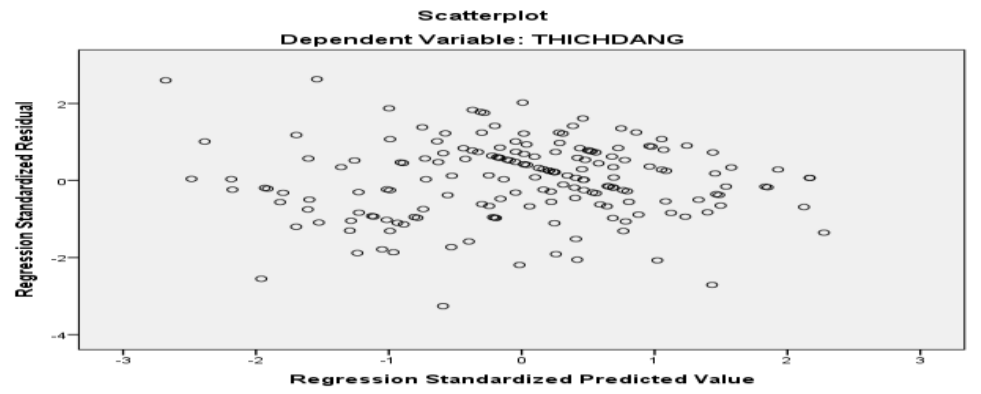

Hình 2: Biểu đồ phân tán giữa giữa các phần dư và giá trị dự đoán của Mô hình hồi quy tuyến tính được sử dụng cho nghiên cứu

Nguồn: số liệu khảo sát thực tế 2015 và được kết xuất tù phần mềm SPSS

Kiểm tra giả định phương sai của sai số kiểm định Spearman (Bảng 8) cho thấy giá trị Sig không đổi: tác giả dùng kiểm định tương quan khá cao $(=0,237>5 \%)$. Như vậy giả thuyết này hạng Spearman để kiểm tra giả định này. Kết quả được chấp nhận.

BẢNG 8

KIỂM ĐỊNH SPEARMAN CHO GIẢ ĐỊNH PHƯƠNG SAI CỦA SAI SỐ KHÔNG ĐỔI

\begin{tabular}{|c|c|c|c|c|}
\hline \multicolumn{5}{|c|}{ Tương quan (Correlations) } \\
\hline & & & ABScuare & THICHDANG \\
\hline \multirow[t]{6}{*}{ Spearman's rho } & ABScuare & Hệ số tương quan & 1,000 &,- 089 \\
\hline & & Giá trị Sig. (2-tailed) & . & ,237 \\
\hline & & $\mathrm{N}$ & 180 & 180 \\
\hline & THICHDANG & Hệ số tương quan &,- 089 & 1,000 \\
\hline & & Giá trị Sig. (2-tailed) & ,237 & \\
\hline & & $\mathrm{N}$ & 180 & 180 \\
\hline
\end{tabular}

Nguồn: số liệu khảo sát thực tế 2015 và được kết xuát tì̀ phẩn mềm SPSS.

Kiểm tra giả định về phân phối chuẩn phần dư: nghiên cứu xây dựng Biểu đồ tần suất của các phần dư để kiểm định giả thuyết này. Hình 3 cho thấy một đường cong phân phối chuẩn được đặt chồng lên biểu đồ tần số với phân phối phần dư xấp xỉ chuẩn (trung bình Mean $=0$ và độ lệch chuẩn Std. Dev. $=0,986$ tức là gần bằng 1 ). Điều này cho thấy giả thuyết phân phối chuẩn không bị vi phạm.

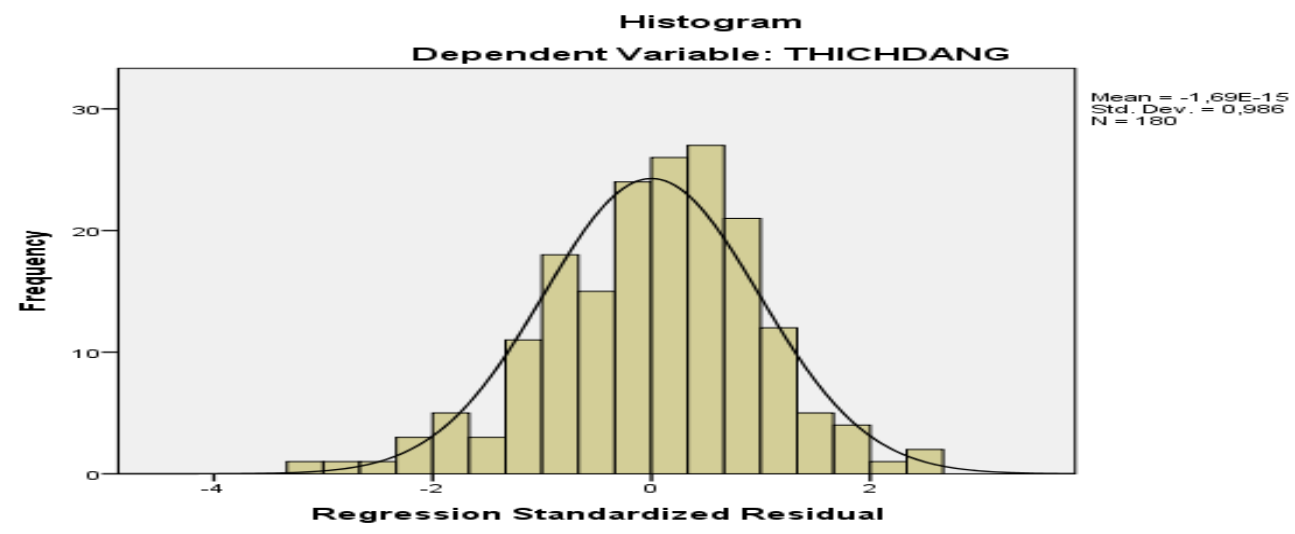

Hình 3: Biểu đồ tần suất của các phần dư chuẩn hóa

(Nguồn: số liệu khảo sát thực tế 2015 và đuợc kết xuất tù phần mềm SPSS) 
Kiểm tra giả định về tính độc lập của sai số: kiểm định Durbin- Watson (Bảng 9) cho thấy giá trị $\mathrm{d}=1,974$ (gần bằng 2), như vậy giả định về tính độc lập của sai số được thỏa mãn.

BẢNG 9

KẾT QUẢ KIỂM ĐỊNH DURBIN- WATSON

\begin{tabular}{c|c|c|c|c|c}
\hline \hline \multicolumn{7}{c}{ Tóm tắt mô hình (Model Summary } \\
\hline \multirow{3}{*}{ Mô hình } & $\mathrm{R}$ & $\begin{array}{c}\text { R bình } \\
\text { phương }\end{array}$ & $\begin{array}{c}\text { R bình phương hiệu } \\
\text { chỉnh }\end{array}$ & $\begin{array}{c}\text { Sai số chuẩn } \\
\text { (Std. Error of the Estimate) }\end{array}$ & $\begin{array}{c}\text { Giá trị } \\
\text { Durbin-Watson }\end{array}$ \\
1 &, $676^{\mathrm{a}}$ &, 457 &, 442 &, 62940 & 1,974
\end{tabular}

a. Biến độc lập: (Hằng số), NLNVKT, HQKSNB, HTNQT, QTLN, DTvBD b.Biến phụ thuộc: THICHDANG

Nguồn: số liệu khảo sát thực tế 2015 và được kết xuất tì̀ phần mềm SPSS.

Mô hình hồi quy và kiểm định giả thuyết nghiên cứu

Bằng phương pháp đồng thời khi phân tích hồi quy và phương pháp bình phương bé nhất OLS, kết quả phân tích cho thấy hệ số xác định
$\mathrm{R} 2=0,457$ và $\mathrm{R} 2 \mathrm{adj}=0,442$ (Bảng 9). Kiểm định F (Bảng 10) cho thấy mức ý nghĩa $=0,000$. Như vậy, mô hình hồi quy phù hợp. Các biến độc lập giải thích được đến $44,2 \%$ biển thiên của biến phụ thuộc.

BẢNG 10

KÊT QUẢ KIỂM ĐỊNH F

\begin{tabular}{rrrrrrr}
\hline \hline \multicolumn{7}{c}{ ANOVA $^{\mathrm{a}}$} \\
\hline \multicolumn{1}{c}{ Mô hình } & Tổng bình phương & df & Trung bình bình phương & F & Giá trị Sig. \\
\hline 1 & Hồi quy & 58,079 & 5 & 11,616 & 29,322 &, $000^{\mathrm{b}}$ \\
& Phần dư & 68,930 & 174 &, 396 & & \\
& Tổng cộng & 127,009 & 179 & & & \\
\end{tabular}

a. Dependent Variable: THICHDANG $\quad$ b. Predictors: (Constant), NLNVKT, HQKSNB, HTNQT, QTLN, DTvBD

Nguồn: số liệu khảo sát thục tế 2015 và được kết xuất tù̀ phần mềm SPSS.

BẢNG 11

BẢNG TRỌNG SỐ HỒI QUY

\begin{tabular}{|c|c|c|c|c|c|c|c|c|}
\hline \multicolumn{9}{|c|}{ Coefficients $^{\mathrm{a}}$} \\
\hline & & \multicolumn{2}{|c|}{ Hệ số chưa chuẩn hóa } & \multirow{2}{*}{$\begin{array}{c}\text { Hệ số chuẩn hóa } \\
\text { Beta }\end{array}$} & \multirow{2}{*}{$\begin{array}{c}\text { Giá trị } \\
\mathrm{t}\end{array}$} & \multirow{2}{*}{$\begin{array}{c}\text { Mức ý nghĩa } \\
\text { Sig. }\end{array}$} & \multicolumn{2}{|c|}{ Thống kê cộng tuyến } \\
\hline \multicolumn{2}{|c|}{ Mô hình } & B & Std. Error & & & & Dung sai & VIF \\
\hline \multirow[t]{6}{*}{1} & (Constant) & 1,759 & ,440 & & 3,993 &, 000 & & \\
\hline & QTLN &,- 254 &, 056 &,- 294 & $-4,535$ &, 000 &, 744 & 1,344 \\
\hline & HTNQT & ,295 & ,081 & ,242 & 3,649 & ,000 & ,709 & 1,410 \\
\hline & ĐTvBD &, 132 &, 070 & ,138 & 1,888 & ,061 &, 586 & 1,706 \\
\hline & HQKSNB &, 105 & ,069 &, 112 & 1,513 &, 132 &, 572 & 1,749 \\
\hline & NLNVKT &, 184 & ,061 & ,193 & 3,030 &, 003 & ,769 & 1,301 \\
\hline
\end{tabular}

a. Dependent Variable: THICHDANG

Nguồn: số liệu khảo sát thục tế 2015 và được kết xuất tù̀ phần mềm SPSS

Kết quả kiểm định các giả thuyết nghiên cứu
Dựa vào kết quả ở bảng trọng số hồi quy, kết quả kiểm định các giả thuyết nghiên cứu được trình bày như sau (Bảng 12): 
BẢNG 12. KÊT QUẢ KIỄM ĐỊNH CÁC GIẢ THUYÊT NGHIÊN CỨU

\begin{tabular}{lll}
\hline \hline \multicolumn{1}{c}{ Giả thuyết } & Kết quả & \multicolumn{1}{c}{ Kết luận } \\
\hline $\begin{array}{l}\text { H1: QTLN tác động ngược chiều đến tính } \\
\text { Thích đáng của CLTT BCTC }\end{array}$ & Chấp nhận & $\begin{array}{l}\text { Thích đáng của BCTC sẽ giảm đi } 0,294 \text { điểm tương ứng với } \\
\left.\text { mồi điểm tăng thêm của QTLN } *^{*}\right)\end{array}$ \\
\hline $\begin{array}{l}\text { H2: HTNQT tác động cùng chiều đến } \\
\text { CLTT BCTC. }\end{array}$ & Chấp nhận & $\begin{array}{l}\text { Thích đáng của BCTC sẽ tăng thêm 0,242 điểm tương ứng với } \\
\text { mỗi điểm tăng thêm của HTNQT }\left(^{*}\right)\end{array}$ \\
\hline $\begin{array}{l}\text { H3: ĐTvBD có động cùng chiều đến CLTT } \\
\text { BCTC }\end{array}$ & Chấp nhận & $\begin{array}{l}\text { Thích đáng của BCTC sẽ tăng thêm } 0,138 \text { điểm tương ứng với } \\
\text { mỗi điểm tăng thêm của ĐTvBD }(* *)\end{array}$ \\
\hline $\begin{array}{l}\text { H4: HQKSNB tác động đến cùng chiều } \\
\text { CLTT BCTC }\end{array}$ & Bác bỏ & $\begin{array}{l}\text { Chưa có bằng chứng cho thấy HQKSNB tác động đến tính } \\
\text { Thích đáng của BCTC }\end{array}$ \\
\hline $\begin{array}{l}\text { H5: NLNVKT tác động đến cùng chiều } \\
\text { CLTT BCTC }\end{array}$ & Chấp nhận & $\begin{array}{l}\text { Thích đáng của BCTC sẽ tăng thêm } 0,193 \text { điểm tương ứng với } \\
\text { mồi điểm tăng thêm của NLNVKT (*) }\end{array}$ \\
\hline \hline
\end{tabular}

Ghi chú: (*): múc ý nghĩa thống kê = 5\%; (**): múc ý nghĩa thống kê =10\%

\section{Mô hình hồi quy}

Bảng 11 cho thấy VIF của các biến đều rất thấp $(<2)$, vì vậy hiện tượng đa cộng tuyến không xảy ra. Dựa vào trọng số hồi quy chuẩn hóa $(\beta)$, mô hình hồi quy tuyến tính được rút ra như sau:

\begin{tabular}{|ll|}
\hline THICHDANG $=1,759-0,294 \mathrm{QTLN}+0,242 \mathrm{HTNQT}+0,138 \mathrm{DTVBD}+0,193 \mathrm{NLNVKT}$ \\
\hline
\end{tabular}

Trong đó: THICHDANG: tính Thích đáng của CLTT BCTC; QTLN: Hành vi quản trị lợi nhuận; HTNQT: Hỗ trợ từ phía nhà quản trị; ĐTvBD: Đào tạo và bồi dưỡng; NLNVKT: Năng lực nhân viên kế toán.

Mô hình hồi quy tuyến tính từ SPSS cho thấy có tổng cộng có 4 nhân tố tác động đến tính Thích đáng của CLTT BCTC, trong đó, QTLN có tác động ngược chiều và 3 nhân tố còn lại có tác động thuận chiều. Trong số các nhân tố tác động, nhân tố QTLN có tác động mạnh nhất, điều này cho thấy tác động từ phía nhà quản trị có ảnh hưởng mạnh mẽ đến tính Thích đánh của CLTT BCTC trong các $\mathrm{DN}$ hiện nay. Bên cạnh đó, chưa có bằng chứng cho thấy $\mathrm{HQKSNB}$ có tác động đến tính Thích đáng của CLTT BCTC ở mức có ý nghĩa thống kê.

\section{KÊT LUẬN VÀ GỢI Ý CHÍNH SÁCH}

\subsection{Kết luận}

Kết quả nghiên cứu cho thấy yêu cầu Thích đáng của CLTT BCTC trong các DN Việt Nam hiện nay được đánh giá ở mức độ chấp nhận được được với số điểm là 3,72 . Trong 2 thành phần cấu thành nên thuộc tính này, thành phần Giá trị xác nhận được đánh giá ở mức điểm bình quân là 3,73 và Giá trị dự đoán là 3,71 . Điều này cho thấy yêu cầu Thích đáng của $\mathrm{BCTC}$ cần phải được xem xét và cải thiện.

Với hệ số xác định $\mathrm{R} 2 \mathrm{adj}=0,442$, nghiên cứu giúp khẳng định tầm quan trọng của các nhân tố bên trong DN khi nó giải thích được đến 44,2\% biến thiên của CLTT BCTC xét trên khía cạnh Thích đáng. Bằng mô hình hồi quy, nghiên cứu đã giúp lượng hóa, đo lường mức độ tác động của các nhân tố QTLN; HTNQT; ĐTvBD và NLNVKT đến tính Thích đáng của CLTT BCTC.

\subsection{Gơi ý chính sách}

Từ kết quả nghiên cứu trên, tác giả đề xuất một số khuyến nghị nhằm nâng cao chất lượng thông tin BCTC của các DN Việt Nam như sau: cần thực hiện các giải pháp hạn chế tác động tiêu cực của QTLN thông qua viêc thực hiện một cơ chế giám sát từ phía bên trong lẫn bên ngoài $\mathrm{DN}$ cũng như xây dựng cơ chế động viên khen thưởng hợp lý; DN cần xây dựng chiến lược phát triển hệ thống thông tin phù hợp với chiến lược kinh doanh của toàn $\mathrm{DN}$, phổ biến và truyền thông một cách đầy đủ và hiệu quả chiến lược này cho tất cả các bộ phận trong $\mathrm{DN}$; DN cần có chính sách hỗ trợ, khuyến khích nhân viên của mình thường xuyên cập nhật các quy định, chính sách về $\mathrm{KT}$, 
thuế; Các cơ sở đào tạo, đội ngũ giảng viên và Hội kế toán và kiểm toán Việt Nam (VAA) cần thể hiện vai trò và trách nhiệm của mình trong việc cải thiện CL nguồn nhân lực kế toán và kiểm toán.

\subsection{Hạn chế và hướng nghiên cứu kế tiếp}

Theo FASB \& IASB, các thuộc tính cấu thành nên CLTT BCTC rất đa dạng [7]. Bên cạnh thuộc tính Thích đáng, nghiên cứu kế tiếp cần tiếp cận CLTT BCTC ở khía cạnh Trình bày trung thực (Faithful representation) hoặc thuộc tính gia tăng CLTT BCTC hoặc trên cả ba khía cạnh này. Bên cạnh đó, nghiên cứu cũng cần xây dưng mô hình hồi quy bao gồm cả các nhân tố bên trong lẫn bên ngoài DN.

\section{TÀI LIỆU THAM KHẢO}

[1]Céline Michailesco (2010). Qualité de l'information comptable, Encyclopédie de Comptabilité. Contrôle de gestion et Audit Economica, pp. 1023 - 1033, 2010 <halshs$00540571>$.

[2]FASB, 1993. Statement of Financial Accounting Concepts No.2, Qualitative Characteristics of Accounting Information.
Financial Accounting Standards Board, http://www.fasb.org/jsp/FASB/Page/PreCodSectionPage\&cid= 1176156317989 truy cập ngày 15/3/2014.

[3] Geert Braam \& Ferdy van Beest, 2013. Conceptually-Based Financial Reporting Quality Assessment. An Empirical Analysis on Quality Differences Between UK Annual Reports and US 10-K Reports. NiCE Working Paper 13-106 November 2013

[4]Gelinas, Sutton \& Oram (1999). Accounting Information Syatem. South- Western.

[5]Hongjiang $\mathrm{Xu}$ (2003). Critical success factors for accounting information systems data quality. PhD Thesis, University of Southern Queesland.

[6]IASB (2001). Framework for the preparation and presentation of financial statements. IFRS, http://www.ifrs.org/IFRSs/Documents/ConceptualFramework. pdf truy cập ngày 15/03/2014

[7]IASB (2010). Conteptual Framework for Financial Reporting 2010. IFRS Foundation.

[8]James A. Hall (2011). Accounting Information Syatem. South- Western.

[9] Joseph P. H. Fan \& T.J. Wong (2002). Corporate ownership structure and the informativeness of accounting earnings in East Asia. Journal of Accounting and Economies, Vol 33, pp 401- 425 .

[10] Nguyễn Đình Thọ (2011). Phương pháp nghiên cứu khoa học trong kinh doanh. NXB Lao động- Xã hội.

\title{
Impact of internal factors on the financial statements information quality of enterprises in Vietnam
}

\author{
Pham Quoc Thuan \\ University of Economics and Law, VNUHCM, Viet Nam \\ Corresponding author: thuanpq@,uel.edu.vn
}

Received: 7-02-2018, Accepted: 02-5-2018; Published: 15-7-2018

\begin{abstract}
This research aims to construct scales to measure the quality of financial statement information directly based on sets of characteristics of financial statements quality, published by FASB $\&$ IASB 2010. Additionally, based on qualitative research, the study identifies five internal factors affecting the relevance of financial statements information quality, including: earnings management behaviors, top management support,
\end{abstract}

education and training, internal control system efficiency and accountants' competencies. Based on quantitative research, the study constructed regression model showing the influence of $\mathbf{4}$ factors, namely earnings managements, top management support, education and training, and accountants competencies om the relevance of financial statements information quality.

Keywords-Relevance, information quality, financial statement, earnings managements, top management support, education and training, internal control system efficiency, accountant's competencies. 